Case Report

\title{
Brevibacterium Bacteremia in the Setting of Pyogenic Liver Abscess: A Case Report with Accompanying Literature Review
}

\author{
Sarah Hossain $\mathbb{D}^{1},{ }^{1}$ Afif Hossain $\mathbb{D},{ }^{2}$ Aldo Barajas-Ochoa $\mathbb{D}^{2},{ }^{2}$ and Michael A. Jaker ${ }^{2}$ \\ ${ }^{1}$ American University of Antigua, Jabberwock Beach Road, PO Box W1451, Coolidge, Antigua and Barbuda \\ ${ }^{2}$ Department of Internal Medicine, Rutgers New Jersey Medical School, 185 S Orange Avenue, Newark, NJ 07103, USA \\ Correspondence should be addressed to Afif Hossain; hossaina@njms.rutgers.edu
}

Received 22 August 2021; Revised 14 December 2021; Accepted 15 December 2021; Published 28 December 2021

Academic Editor: Larry M. Bush

Copyright (C) 2021 Sarah Hossain et al. This is an open access article distributed under the Creative Commons Attribution License, which permits unrestricted use, distribution, and reproduction in any medium, provided the original work is properly cited.

A 71-year-old Pakistani man with poorly controlled type 2 diabetes mellitus presenting with worsening mental status, abdominal pain, and oral intake for the past seven days was found to have pyogenic hepatic abscess with unculturable bacteria and subsequently found to have rare Brevibacterium bacteremia.

\section{Introduction}

Brevibacterium is a short coryneform species found in dairy products and known colonizers of the human skin. Early in its life cycle, Brevibacterium exhibits typical features of coryneform bacteria. However, as it matures, it takes on an appearance similar to cocci or coccobacilli [1]. The most common species isolated from humans is Brevibacterium casei [2], which appears as a catalase-positive, non-sporeforming, short, club-shaped rod on gram staining. Most commonly, bacteremia is associated with indwelling intravascular catheters in the immunocompromised [3]. However, there are rare cases leading to meningitis, cholangitis, salpingitis, peritonitis, endocarditis, and osteomyelitis. The treatment of choice for serious infections is vancomycin as the bacteria show some resistance to B-lactams, fluoroquinolones, clindamycin, and macrolide antibiotics [4]. To date, there are only 16 published case reports of Brevibacterium bacteremia [5].

\section{Case Presentation}

A 71-year-old Pakistani man presented to University Hospital in Newark, NJ, in May of 2020 after being brought in by emergency medical services from home for worsening mental status over the last seven days. The patient had a history of triple vessel coronary artery disease after percutaneous coronary intervention and stenting to the acute marginal branch of the right coronary artery in June, 2018, balloon angioplasty to the proximal and middle right coronary artery in 2011, and stenting of the left anterior descending artery in 2011, poorly controlled type 2 diabetes mellitus, hypertension, and hyperlipidemia. According to the patient's family, he had worsening mental status, decreased oral intake, and worsening abdominal pain over the last week, with a sharp decline over the past two days.

At baseline, the patient was able to perform all activities of daily living independently, was able to hold full conversations in English and Urdu, and had no issues with ambulation. He had no other symptoms prior to arrival. Furthermore, the patient was not taking his medications regularly, including insulin. For diabetes, the patient was previously prescribed insulin, metformin, glimepiride, canagliflozin, and sitagliptin. However, it was unclear which of these medications he was indeed taking as he had not been seen outpatient in over 10 years. Of note, the patient had traveled to Pakistan and Dubai within the last year but did not have any sick contacts.

In the emergency room, the patient was found to be febrile to $101.8^{\circ} \mathrm{F}$, tachycardic to 128 , normotensive, tachypneic to 20 , and saturating $99 \%$ on room air. On exam, he was disoriented and confused, speaking incoherently in one-word sentences, had poor dentition with dry mucous membranes, tachycardic, and had diffuse abdominal 
tenderness to minimal palpation, worse in the right upper quadrant but without rebound or guarding. Admission labs were significant for a leukocytosis of 12 , hemoglobin of 8.3 from a baseline of 12 with a mean corpuscular volume of 63.1 , sodium of 124, bicarbonate of 18, glucose of 399 but negative acetone, anion gap of 18 with lactic acidosis to 3.5 , HbAlc 12.2, procalcitonin 8.16, c-reactive protein 50, negative cardiac enzymes, and normal liver enzymes. Given his altered mental status and severe sepsis, the patient had computed tomography of the abdomen and pelvis with intravenous contrast carried out (Figure 1), showing multiple colonic diverticula without diverticulitis and a 6 by 4.6 centimeter rim-enhancing lesion with surrounding satellite lesions, likely representing a liver abscess.

The patient was started on aggressive intravenous fluid resuscitation, morphine for pain control, and intravenous Flagyl (metronidazole) and cefepime for 2 days. Vancomycin was later added to cover for Enterococcus but discontinued after three doses, so no level was checked. COVID-19 testing, interferon- $\gamma$ release assay, Entamoeba histolytica, and Echinococcus serologies were all negative. During his hospital course, the patient had uncontrollable blood sugars and ultimately required 65 units of Lantus daily, 8 units of lispro with meals, and an additional 20-25 units of coverage daily. Abscess drainage by interventional radiology was performed on admission and then again 6 days later. Two sets of body fluid cultures were sent, which grew Gram-positive cocci in pairs and chains but could not be speciated. Blood cultures eventually speciated Brevibacterium from aerobic bottles twice. Unfortunately, realtime polymerase chain reaction was unable to identify speciation for the causative organism for either set of body fluid cultures or blood cultures. Infectious Disease was consulted, and the patient was switched to ampicillin-sulbactam (Unasyn) $3 \mathrm{~g}$ every six hours for a minimum 3-week course with a plan for repeat imaging after 3 weeks of intravenous antibiotics. After a 10-day hospital stay and improvement in his condition, the patient was safely transferred to subacute rehabilitation for continued antibiotic therapy.

\section{Discussion and Literature Review}

To date, there are only 18 publications mentioning Brevibacterium bacteremia, with 16 of those cases published as case reports [5]. The earliest publication, dating back to 1973, involved pediatric patients undergoing carious extraction for moderate to severe dental pathology [6]. Blood cultures were drawn several times pre- and postoperatively, with one of thirty-four patients testing positive for Brevibacterium. In Saudi Arabia, a 2004 study found Brevibacterium bacteremia in four critically ill patients, highlighting its involvement in serious infections in the immunocompromised [7]. From the published case reports, 11 speciated Brevibacterium casei, 1 Brevibacterium epidermidis, 1 Brevibacterium paucivorans, 1 Brevibacterium massiliense, and 2 were not specified. Seven patients had underlying malignancy, two had AIDS, five had chronic medical comorbidities, one had a congenital abnormality of

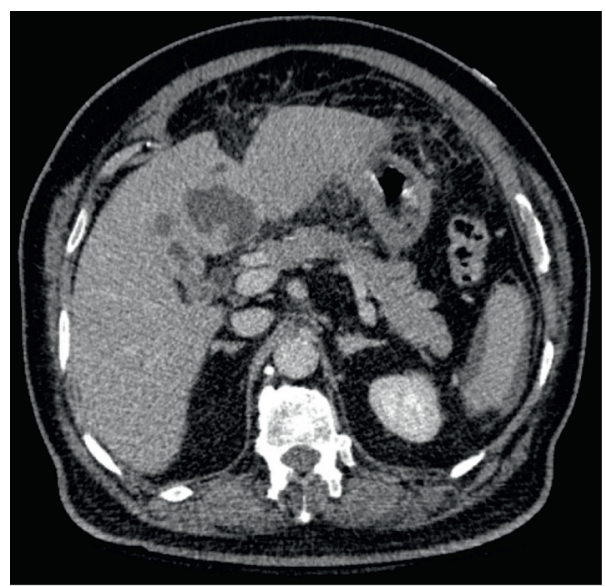

FIgURE 1: Computed tomography of the abdomen and pelvis with intravenous contrast in the transverse section showing rim-enhancing fluid-filled collections in hepatic segments $4 \mathrm{~A}$ and $4 \mathrm{~B}, 6 \mathrm{~cm}$ by $4.6 \mathrm{~cm}$ in size in the greatest axial dimensions. There are several smaller, localized satellite lesions. Findings are highly suspicious for a liver abscess.

metabolism, and one was not mentioned. 13 patients had some form of indwelling catheter, 2 were not specified, and 1 had no indwelling catheter present. The majority of patients were given broad-spectrum antibiotics, including vancomycin, teicoplanin, aminoglycosides, extended-spectrum beta lactams, and/or fluoroquinolones. 14 patients improved, 6 patients had recurrence, and 2 patients died. See Table 1 for further details.

As mentioned previously, Brevibacterium are nonmotile, non-spore-forming, non-acid-fast, obligate aerobes. They are Gram-positive bacilli in singlets, pairs, or short chains which transform into cocci approximately 48 hours after inoculation. Characteristic features include positivity for lipase, catalase, DNAse, litmus milk, oxidase variability, urease negativity, and reduction of nitrates to nitrites [1]. Though there are various strains, Brevibacterium casei is the most common clinically relevant pathogen [2]. Brevibacterium species are typically innocuous bacteria found in cheeses, poultry, and human skin flora. However, in the immunocompromised and, especially, those with indwelling catheterization [3], they can function as devastating opportunistic pathogens. In addition to the aforementioned bacteremia cases, they rarely can cause brain abscess, meningitis, endocarditis, pericarditis, peritonitis, cholangitis, salpingitis, mastitis, and osteomyelitis. Although vancomycin is used most frequently, ultimately the antibiotic chosen depends on the infection site and severity of infection [4].

In this case report, a patient with pyogenic liver abscess was found to have concomitant Brevibacterium bacteremia. To our knowledge, this is the first instance of such a phenomenon and one of the only cases without central-lineassociated infection. It is unclear if our patient had bacterial translocation from colonic diverticula leading to hepatic abscess. Another potential source is yogurt and cheese consumed by the patient while abroad in Pakistan and/or Dubai, which is a common delicacy with most meals. Despite 
TABLE 1: Clinical summaries of Brevibacterium bacteremia case reports.

\begin{tabular}{|c|c|c|c|c|c|c|c|c|}
\hline $\begin{array}{l}\text { Author } \\
\text { (year) }\end{array}$ & Sex & Age & $\begin{array}{l}\text { Brevibacterium } \\
\text { species }\end{array}$ & Underlying condition & Clinical course & $\begin{array}{l}\text { Treatment } \\
\text { regimen } \\
\text { (duration) } \\
\end{array}$ & $\begin{array}{c}\text { Presence of an } \\
\text { indwelling } \\
\text { catheter }\end{array}$ & Outcome \\
\hline $\begin{array}{l}\text { McCaughey } \\
\text { and Damani } \\
(1991)[8]\end{array}$ & M & 40 & epidermidis & $\begin{array}{l}\text { Zollinger-Ellison } \\
\text { Syndrome }\end{array}$ & $\begin{array}{l}\text { Vomiting, weight } \\
\text { loss, recurrent } \\
\text { duodenal } \\
\text { ulceration, pyloric } \\
\text { outflow obstruction }\end{array}$ & $\begin{array}{l}\text { Erythromycin, } \\
\text { TLC }^{\mathrm{a}} \text { removal }\end{array}$ & $\begin{array}{l}\text { Yes- indwelling } \\
\text { subclavian } \\
\text { TLC }^{\mathrm{a}} \text { for } \mathrm{TPN}^{\mathrm{b}}\end{array}$ & Survived \\
\hline $\begin{array}{l}\text { Lina et al. } \\
(1994)[9]\end{array}$ & $\mathrm{M}$ & 19 & Not specified & $\begin{array}{l}\text { Lymphoblastic } \\
\text { Lymphoma }\end{array}$ & $\begin{array}{l}\text { Fever, retroocular } \\
\text { pain; recurrence } \\
\text { after } 1 \text { month }\end{array}$ & $\begin{array}{l}\text { IV teicoplanin, } \\
\text { amikacin for } \\
20 \text { days; } \\
\text { teicoplanin } \\
\text { x21 days, TLC } \\
\text { removal }\end{array}$ & $\begin{array}{l}\text { Yes- for } \\
\text { chemotherapy; } \\
\text { type not } \\
\text { specified }\end{array}$ & $\begin{array}{l}\text { Recurrence; } \\
\text { survived }\end{array}$ \\
\hline $\begin{array}{l}\text { Reinert et al. } \\
\text { (1995) [10] }\end{array}$ & M & 25 & casei & $\begin{array}{c}\text { Testicular } \\
\text { choriocarcinoma }\end{array}$ & Fever, pancytopenia & $\begin{array}{l}\text { IV piperacillin, } \\
\text { teicoplanin for } \\
10 \text { days; } \\
\text { piperacillin, } \\
\text { tobramycin for } \\
10 \text { days }\end{array}$ & $\begin{array}{l}\text { Yes- } \text { TLC }^{\mathrm{a}} \text { for } \\
\text { chemotherapy }\end{array}$ & $\begin{array}{l}\text { Recurrence; } \\
\text { survived }\end{array}$ \\
\hline $\begin{array}{l}\text { Kaukoranta- } \\
\text { Tolvanen } \\
\text { et al. (1995) } \\
\text { [11] }\end{array}$ & $\mathrm{F}$ & 56 & casei & $\begin{array}{l}\text { Non-Hodgkin } \\
\text { lymphoma }\end{array}$ & $\begin{array}{c}\text { Fever, } \\
\text { pancytopenia, CRP } \\
42 \mathrm{mg} / \mathrm{dL}\end{array}$ & Not specified & $\begin{array}{l}\text { Yes- TLC }{ }^{\mathrm{a}} \text { for } \\
\text { chemotherapy }\end{array}$ & $\begin{array}{l}\text { Recurrence; } \\
\text { survived }\end{array}$ \\
\hline $\begin{array}{l}\text { Castagnola } \\
\text { et al. (1997) } \\
{[12]}\end{array}$ & --- & --- & casei & Neuroblastoma & $\begin{array}{c}\text { Fever, } \\
\mathrm{ANC}^{\mathrm{d}}>1000 \mathrm{~cm}^{3}\end{array}$ & $\begin{array}{l}\text { Not specified; } \\
\text { TLC }{ }^{\text {a }} \text { removed }\end{array}$ & $\begin{array}{l}\text { Yes- Broviac }{ }^{\circledR} \\
\text { for } \\
\text { chemotherapy }\end{array}$ & Survived \\
\hline $\begin{array}{l}\text { Brazzola } \\
\text { et al. (2000) } \\
{[13]}\end{array}$ & $\mathrm{F}$ & 18 & casei & $\begin{array}{c}\text { Acquired } \\
\text { Immunodeficiency } \\
\text { Syndrome (AIDS) }\end{array}$ & Fever, dehydration & $\begin{array}{l}\text { IV Unasyn, } \\
\text { switched to } \\
\text { ciprofloxacin } \\
\text { for } 14 \text { days; } \\
\text { TLC }^{\text {a }} \text { removed }\end{array}$ & $\begin{array}{c}\text { Yes- Port-A } \\
\text { Cath }^{\circledR} \text { for PPN }\end{array}$ & Survived \\
\hline $\begin{array}{l}\text { Ogunc et al. } \\
(2002)[14]\end{array}$ & --- & 60 & Not specified & $\begin{array}{l}\text { Chronic Lymphocytic } \\
\text { Leukemia (CLL) }\end{array}$ & $\begin{array}{c}\text { Fever following } \\
\text { fludarabine } \\
\text { chemotherapy, } \\
\text { anemia }\end{array}$ & $\begin{array}{l}\text { IV ceftazidime, } \\
\text { amikacin; } \\
\text { switched to } \\
\text { vancomycin }\end{array}$ & Not specified & Survived \\
\hline $\begin{array}{l}\text { Janda et al. } \\
\text { (2003) [7] }\end{array}$ & M & 34 & casei & $\begin{array}{c}\text { Acquired } \\
\text { Immunodeficiency } \\
\text { Syndrome (AIDS) }\end{array}$ & $\begin{array}{l}\text { CD4<50, known } \\
\text { CMV retinitis, } \\
\text { oropharyngeal } \\
\text { candidiasis, } \\
\text { neutropenic fever, } \\
\text { malaise }\end{array}$ & $\begin{array}{l}\text { IV vancomycin } \\
\text { for } 8 \text { days, } \\
\text { ceftazidime } \\
\text { (stopped); } \\
\text { TLC }^{\text {a }} \text { removed }\end{array}$ & $\begin{array}{l}\text { Yes- Hickman }{ }^{\circledR} \\
\text { catheter for } \\
\text { long-term } \\
\text { gancylovir } \\
\text { infusion }\end{array}$ & Survived \\
\hline $\begin{array}{l}\text { Beukinga } \\
\text { et al. (2004) } \\
{[15]}\end{array}$ & $\mathrm{F}$ & 43 & casei & Crohn's Disease & $\begin{array}{l}\text { Chronic fistulae, } \\
\text { total colectomy, } \\
\text { fever, WBC } \text { WB }^{\mathrm{f}} 3300 \text {, } \\
\mathrm{CRP}^{\mathrm{c}} 5.8 \mathrm{mg} / \mathrm{dL}\end{array}$ & $\begin{array}{l}\text { IV vancomycin } \\
\text { for } 15 \text { days, } \\
\text { TLC }{ }^{a} \text { remained; } \\
\text { IV Unasyn, } \\
\text { Merrem, } \\
\text { amikacin, TLC } \\
\text { removed }\end{array}$ & $\begin{array}{c}\text { Yes- Port-A } \\
\text { Cath }^{\circledR} \text { for } \mathrm{PPN}^{\mathrm{e}}\end{array}$ & $\begin{array}{c}\text { Recurrence } \\
\text { (at } \\
5 \text { months); } \\
\text { died }\end{array}$ \\
\hline $\begin{array}{l}\text { Beukinga } \\
\text { et al. (2004) } \\
{[15]}\end{array}$ & M & 31 & casei & Not specified & $\begin{array}{c}\text { Fever, } \mathrm{WBC}^{\mathrm{f}} 4700, \\
\operatorname{CRP}^{\mathrm{c}}(-)\end{array}$ & $\begin{array}{l}\text { IV vancomycin } \\
\text { for } 15 \text { days, } \\
\text { TLC remained; } \\
\text { same treatment }\end{array}$ & $\begin{array}{l}\text { Yes- Hickman }{ }^{\circledR} \\
\text { catheter for } \\
\text { hemodialysis }\end{array}$ & $\begin{array}{l}\text { Recurrence } \\
\quad \text { (at } \\
5 \text { months); } \\
\text { survived }\end{array}$ \\
\hline $\begin{array}{l}\text { Ulrich et al. } \\
(2006) \text { [3] }\end{array}$ & $\mathrm{F}$ & 62 & casei & $\begin{array}{l}\text { Severe pulmonary } \\
\text { hypertension }\end{array}$ & $\begin{array}{l}\text { Flu-like symptoms, } \\
\text { productive cough, } \\
\text { chills, fever, } \\
\text { hypoxemia, CRP } \\
38 \mathrm{mg} / \mathrm{dL}\end{array}$ & $\begin{array}{l}\text { IV vancomycin } \\
\text { for } 10 \text { days, } \\
\text { then } \\
\text { moxifloxacin } \\
\text { for } 20 \text { days, } \\
\text { TLC }^{\text {a }} \text { removed }\end{array}$ & $\begin{array}{l}\text { Yes- TLC }{ }^{\mathrm{a}} \text { for } \\
\text { iloprost } \\
\text { infusion }\end{array}$ & Survived \\
\hline
\end{tabular}


TABLE 1: Continued.

\begin{tabular}{|c|c|c|c|c|c|c|c|c|}
\hline $\begin{array}{l}\text { Author } \\
\text { (year) }\end{array}$ & Sex & Age & $\begin{array}{l}\text { Brevibacterium } \\
\text { species }\end{array}$ & Underlying condition & Clinical course & $\begin{array}{l}\text { Treatment } \\
\text { regimen } \\
\text { (duration) } \\
\end{array}$ & $\begin{array}{l}\text { Presence of an } \\
\text { indwelling } \\
\text { catheter }\end{array}$ & Outcome \\
\hline $\begin{array}{l}\text { Bal et al. } \\
(2015)[16]\end{array}$ & M & 6 & casei & $\begin{array}{c}\text { Acute Lymphoblastic } \\
\text { Leukemia (ALL), B } \\
\text { cell type }\end{array}$ & $\begin{array}{c}\text { Herpes zoster } \\
\text { infection, } \\
\text { pancytopenia, } \\
\text { neutropenic fever, } \\
\text { ANC }^{\mathrm{d}} 387 \mathrm{~mm} 3 / \mathrm{uL}, \\
\mathrm{CRP}^{\mathrm{c}} 6.1 \mathrm{mg} / \mathrm{dL}\end{array}$ & $\begin{array}{l}\text { IV Zosyn, } \\
\text { vancomycin for } \\
10 \text { days, TLC } \\
\text { remained }\end{array}$ & $\begin{array}{l}\text { Yes- Hickman }{ }^{\circledR} \\
\text { catheter for } \\
\text { chemotherapy }\end{array}$ & Survived \\
\hline $\begin{array}{l}\text { Bonavila } \\
\text { Juan et al. } \\
(2017) \text { [17] }\end{array}$ & M & 60 & casei & $\begin{array}{c}\text { Child-Pugh C } \\
\text { alcoholic cirrhosis, } \\
\text { aortic stenosis; } \\
\text { development of aortic } \\
\text { valve endocarditis and } \\
\text { insufficiency with } \\
\text { recurrence }\end{array}$ & $\begin{array}{c}\text { Tremor, altered } \\
\text { mental status, fever, } \\
\text { pustular rash; } \\
\text { decompensated } \\
\text { cirrhosis, } \\
\text { coagulopathy, } \\
\text { thrombocytopenia, } \\
1.5 \mathrm{~cm} \text { aortic valve } \\
\text { vegetation seen on } \\
\text { TEE }^{\mathrm{g}} \text {; right arm } \\
\text { weakness, septic } \\
\text { emboli }\end{array}$ & $\begin{array}{l}\text { Oral Levaquin } \\
\text { for } 10 \text { days, } \\
\text { then } \\
\text { norfloxacin; IV } \\
\text { vancomycin for } \\
4 \text { weeks; IV } \\
\text { vancomycin for } \\
10 \text { days, } \\
\text { daptomycin for } \\
6 \text { days }\end{array}$ & Not specified & $\begin{array}{c}\text { Recurrence } \\
\text { (30 days, } \\
90 \text { days); } \\
\text { died }\end{array}$ \\
\hline $\begin{array}{l}\text { Vecten et al. } \\
(2017)[18]\end{array}$ & M & 4 & massiliense & $\begin{array}{l}\text { Congenital } \\
\text { methylmelonic } \\
\text { acidemia }\end{array}$ & $\begin{array}{c}\text { Fever, cough, } \\
\text { emesis, left ear } \\
\text { discharge, WBC } \mathrm{WB}^{\mathrm{f}} \\
\text { 9400uL, CRP }(-), \\
\text { oxalic acid } \\
0.020 \mathrm{mmol} / \mathrm{L}\end{array}$ & $\begin{array}{l}\text { Intra-auricular } \\
\text { ofloxacin for } \\
8 \text { days }\end{array}$ & $\begin{array}{l}\text { Yes- } \\
\text { gastrostomy } \\
\text { tube present }\end{array}$ & Survived \\
\hline $\begin{array}{l}\text { Magi et al. } \\
\text { (2018) [19] }\end{array}$ & $\mathrm{F}$ & 48 & Casei & $\begin{array}{l}\text { Bilateral breast cancer } \\
\text { requiring } \\
\text { mastectomy, } \\
\text { chemotherapy, } \\
\text { radiation, and } \\
\text { salpingoophorectomy }\end{array}$ & $\begin{array}{l}\text { Fever, myalgia, } \\
\text { CRP }^{c} 5.97 \mathrm{mg} / \mathrm{dL}\end{array}$ & $\begin{array}{l}\text { IV teicoplanin } \\
\text { for } 7 \text { days, } \\
\text { linezolid for } \\
7 \text { days, TLC } \\
\text { removed }\end{array}$ & $\begin{array}{l}\text { Yes- } \\
\text { transjugular } \\
\text { Port-A-Cath } \\
\text { from prior } \\
\text { chemotherapy } \\
\text { treatment }\end{array}$ & Survived \\
\hline $\begin{array}{l}\text { Asai et al. } \\
(2019)[5]\end{array}$ & $\mathrm{F}$ & 94 & paucivorans & $\begin{array}{l}\text { Type } 2 \text { diabetes } \\
\text { mellitus, congestive } \\
\text { heart failure }\end{array}$ & $\begin{array}{l}\text { Fever, decreased } \\
\text { oral intake, appetite } \\
\text { loss, } \\
\text { thrombocytopenia, } \\
\operatorname{CRP}^{\mathrm{c}}(-)\end{array}$ & $\begin{array}{l}\text { IV Merrem, } \\
\text { teicoplanin for } \\
14 \text { days }\end{array}$ & Not present & Survived \\
\hline Our Case & M & 71 & $\begin{array}{l}\text { Unable to } \\
\text { speciate }\end{array}$ & $\begin{array}{l}\text { Poorly controlled type } \\
2 \text { diabetes mellitus, } \\
\text { pyogenic liver abscess }\end{array}$ & $\begin{array}{c}\text { Altered mental } \\
\text { status, abdominal } \\
\text { pain, decreased oral } \\
\text { intake, WBC } \\
\text { 12,000, anemia, } \\
\text { hyponatremia, } \\
\text { hyperglycemia, } \\
\text { HbA1c } 12.2 \text {, acetone } \\
\text { (-), CRP } 50 \mathrm{mg} / \mathrm{dL} \text {, } \\
\text { procalcitonin } 8.16\end{array}$ & $\begin{array}{l}\text { IV Unasyn for } \\
3 \text { weeks, abscess } \\
\text { drainage }\end{array}$ & Not present & Survived \\
\hline
\end{tabular}

$\mathrm{M}=$ male, $\mathrm{F}=$ female; ${ }^{\mathrm{a}} \mathrm{TLC}=$ triple lumen catheter; ${ }^{\mathrm{b}} \mathrm{TPN}=$ total parenteral nutrition, ${ }^{\mathrm{c}} \mathrm{CRP}=\mathrm{c}$-reactive protein, ${ }^{\mathrm{d}} \mathrm{ANC}=$ absolute neutrophil count, ${ }^{\mathrm{e}} \mathrm{PPN}=$ partial parenteral nutrition, ${ }^{\mathrm{f}} \mathrm{WBC}=$ white blood cell, ${ }^{\mathrm{g}} \mathrm{TEE}=$ transesophageal echocardiogram.

very poorly controlled diabetes, the patient showed improvement in his condition after abscess drainage and treatment with intravenous Unasyn.

Despite prior notions that Brevibacterium species pose little to no harm clinically, evolving evidence points towards the contrary. Given the severity of bacteremia cases in the immunosuppressed, Brevibacterium can function as a serious and deadly causative opportunistic agent. Utilization and maintenance of long-term indwelling catheters requires close adherence to sterile technique. Earlier case reports highlight non-specific symptomatology, with an often indolent presentation, which later manifests as florid septicemia. In these cases, prompt initiation of broad-spectrum, empiric antibiotics can be lifesaving but should later be deescalated based on the source of infection and susceptibilities in accordance with proper antibiotic stewardship. 


\section{Conclusions}

Brevibacterium infection is an uncommon but potentially fatal cause of bacteremia in the immunocompromised. This risk is increased with prolonged use of indwelling catheters and implanted devices. Infections are often indolent initially but can rapidly escalate if left untreated. Though initial treatment typically includes broad-spectrum antibiotics, treatment can be de-escalated based on the source of infection and susceptibilities with favorable outcomes. Our report hopes to provide further insight into the management of Brevibacterium bacteremia, specifically in the setting of pyogenic liver abscesses, for which there are no documented cases.

\section{Abbreviations}

COVID-19: Coronavirus disease 2019

M: $\quad$ Male

F: $\quad$ Female

TLC: $\quad$ Triple lumen catheter

TPN: Total parenteral nutrition

CRP: $\quad$ c-reactive protein

ANC: $\quad$ Absolute neutrophil count

PPN: $\quad$ Partial parenteral nutrition

WBC: $\quad$ White blood cell

TEE: Transesophageal echocardiogram.

\section{Data Availability}

No data were used to support this study.

\section{Consent}

Written consent was obtained from the patient for publication of this case report and any accompanying images. A copy of the written consent is available for review by the editor of this journal.

\section{Conflicts of Interest}

The authors declare no conflicts of interest.

\section{Authors' Contributions}

All authors contributed equally to the publication of this case report. All authors read and approved the final manuscript for submission.

\section{Acknowledgments}

The authors thank Dr. Michael Jaker for his insight and guidance in the care of their patient. They thank all the healthcare workers at University Hospital for their assistance in this patient's care and in the publication of this manuscript. Finally, they would also like to acknowledge all those involved in the annual poster presentation at the Rutgers New Jersey Medical School 2021 Research Day, given on 5/ 27/21, the links for which can be found in the following: https://njms.rutgers.edu/departments/medicine/
documents/Research\%20Day\%202021/abstracts/Abstract\% 20-\%20106\%20Hossain.pdf and https://njms.rutgers.edu/ departments/medicine/documents/Research\%20Day\% 202021/posters/Poster\%20presentation\%20-\%20Abstract\% 20106.pdf.

\section{References}

[1] Index, J. E. Bennett, R. Dolin, M. J. Blaser, and D. Mandell, Bennett's Principles and Practice of Infectious Diseases, Elsevier, Amsterdam, Netherlands, Eighth edition, 2015.

[2] E. Gruner, A. G. Steigerwalt, D. G. Hollis et al., "Human infections caused by Brevibacterium casei, formerly CDC groups B-1 and B-3," Journal of Clinical Microbiology, vol. 32, no. 6, pp. 1511-1518, 1994

[3] S. Ulrich, R. Zbinden, M. Pagano, M. Fischler, and R. Speich, "Central venous catheter infection with Brevibacterium sp. in an immunocompetent woman: case report and review of the literature," Infection, vol. 34, no. 2, pp. 103-106, 2006.

[4] H. A. Babay and A. M. Kambal, "Isolation of coryneform bacteria from blood cultures of patients at a University Hospital in Saudi Arabia," Saudi Medical Journal, vol. 25, no. 8, pp. 1073-1079, 2004.

[5] N. Asai, H. Suematsu, A. Yamada et al., "Brevibacterium paucivorans bacteremia: case report and review of the literature," BMC Infectious Diseases, vol. 19, no. 1, p. 344, 2019.

[6] F. A. Berry, S. Yarbrough, N. Yarbrough et al., "Transient bacteremia during dental manipulation in children," Pediatrics, vol. 51, no. 3, pp. 476-479, 1973.

[7] W. Janda, P. Tipirneni, and R. Novak, "Brevibacterium casei bacteremia and line sepsis in a patient with AIDS," Journal of Infection, vol. 46, no. 1, pp. 61-64, 2003.

[8] C. Mccaughey and N. N. Damani, "Central venous line infection caused by Brevibacterium epidermidis," Journal of Infection, vol. 23, no. 2, pp. 211-212, 1991.

[9] B. Lina, A. Carlotti, V. Lesaint et al., "Persistent bacteremia due to Brevibacterium species in an immunocompromised patient," Clinical Infectious Diseases, vol. 18, no. 3, pp. 487-488, 1994.

[10] R. R. Reinert, N. Schnitzler, G. Haase et al., "Recurrent bacteremia due toBrevibacterium casei in an immunocompromised patient," European Journal of Clinical Microbiology \& Infectious Diseases, vol. 14, no. 12, pp. 1082-1085, 1995.

[11] S. S. E. Kaukoranta-Tolvanen, A. Sivonen, A. A. I. Kostiala, P. Hormila, and M. Vaara, "Bacteremia caused byBrevibacterium species in an immunocompromised patient," European Journal of Clinical Microbiology \& Infectious Diseases, vol. 14, no. 9, pp. 801-804, 1995.

[12] E. Castagnola, M. Conte, P. Venzano et al., "Broviac catheterrelated bacteraemias due to unusual pathogens in children with cancer: case reports with literature review," Journal of Infection, vol. 34, no. 3, pp. 215-218, 1997.

[13] P. Brazzola, R. Zbinden, C. Rudin, U. B. Schaad, and U. Heininger, "Brevibacterium casei sepsis in an 18-year-old female with AIDS," Journal of Clinical Microbiology, vol. 38, no. 9, pp. 3513-3514, 2000.

[14] D. Oğünç, M. Gültekin, D. Colak et al., "Bacteremia caused by Brevibacterium species in a patient with chronic lymphocytic leukemia," Haematologia, vol. 32, no. 2, pp. 151-153, 2002.

[15] I. Beukinga, H. Rodriguez-Villalobos, A. Deplano, F. Jacobs, and M. J. Struelens, "Management of long-term catheterrelated Brevibacterium bacteraemia," Clinical Microbiology and Infections, vol. 10, no. 5, pp. 465-467, 2004. 
[16] Z. S. Bal, S. Sen, D. Y. Karapinar, S. Aydemir, and F. Vardar, "The first reported catheter-related Brevibacterium casei bloodstream infection in a child with acute leukemia and review of the literature," Brazilian Journal of Infectious Diseases, vol. 19, no. 2, pp. 213-215, 2015.

[17] C. Bonavila Juan, A. Michelena Bengoechea, B. Zubeltzu Sese, and M. Á. Goenaga Sánchez, "Recurrent endocarditis due to Brevibacterium casei: case presentation and a review of the literature," Enfermedades Infecciosas Y Microbiologia Clinica (English ed.), vol. 35, no. 2, pp. 127-128, 2017.

[18] M. Vecten, F. Gouriet, A. Cano, and D. Raoult, "Brevibacterium massiliense bacteremia," IDCases, vol. 7, no. C, pp. 25-26, 2017.

[19] B. Magi, L. Migliorini, A. Sansoni, and M. G. Cusi, "Brevibacterium casei bacteraemia in a port-a-cath carrier patient: a case report," Infezioni in Medicina, Le, vol. 26, no. 3, pp. 263-265, 2018. 